\title{
Using molecular similarity to construct accurate semiempirical electronic structure theories
}

\author{
Benjamin G. Janesko and David Yaron* \\ Department of Chemistry, Carnegie Mellon University,Pittsburgh, PA 15213
}

\begin{abstract}
Ab initio electronic structure methods give accurate results for small systems, but do not scale well to large systems. Chemical insight tells us that molecular functional groups will behave approximately the same way in all molecules, large or small. This molecular similarity is exploited in semiempirical methods, which couple simple electronic structure theories with parameters for the transferable characteristics of functional groups. We propose that high-level calculations on small molecules provide a rich source of parametrization data. In principle, we can select a functional group, generate a large amount of $a b$ initio data on the group in various small-molecule environments, and "mine" this data to build a sophisticated model for the group's behavior in large environments. This work details such a model for electron correlation: a semiempirical, subsystem-based correlation functional that predicts a subsystem's two-electron density matrix as a functional of its one-electron density matrix. This model is demonstrated on two small systems: chains of linear, minimal-basis $(\mathrm{H}-\mathrm{H})_{5}$, treated as a sum of four overlapping $(\mathrm{H}-\mathrm{H})_{2}$ subsystems; and the aldehyde group of a set of HOC-R molecules. The results provide an initial demonstration of the feasibility of the approach.
\end{abstract}

\footnotetext{
*Electronic address: yaron@cmu.edu
} 


\section{INTRODUCTION}

Canonical ab initio electronic structure methods provide highly accurate electronic structures for small systems of $\mathcal{O}(10)$ atoms. However, these methods are too computationally intensive to apply to large systems. The formal scaling of computational effort for ab initio calculations on an $N$-electron system ranges from $\mathcal{O}\left(N^{3}\right)$ for Hartree theory, to $\mathcal{O}\left(N^{5}\right)$ for $\mathrm{MP} 2$, to $\mathcal{O}\left(e^{N}\right)$ for the exact, full-configuration-interaction (full-CI) solution [1]. Ab initio ("first principles") calculations always begin with a minimal amount of information about the system (e.g. an initial geometry and a basis set), determining practically all of the system's features at runtime.

The computational effort of $a b$ initio calculations can be mitigated using two physicallymotivated approximations: $\mathcal{O}(\mathrm{N})$ and semiempirical approximations.

$\mathcal{O}(\mathrm{N})$ approximations are based on the principle of nearsightedness [2], which states that the interactions between parts of a molecule are largely local in character. (A discussion of nearsightedness can be found in Ref. [3].) $\mathcal{O}(\mathrm{N})$ approximations have been developed for every part of an $a b$ initio calculation, from fast multipole methods for Coulomb effects 4, 5, 6] to divide-and-conquer [7] and other [3, 8, 9] methods for self-consistent field (SCF) calculations, to treatments of electron correlation [10, 11, 12, 13, 14, 15, 16, 17, 18]. A schematic of a nearsightedness-based approximation as outlined in Ref. 2] is shown in Fig. 1].

Semiempirical approximations are based on the principle of molecular similarity: that the properties of atoms and functional groups are largely conserved in different molecules. This principle formalizes the chemical insights that methyl groups are relatively small and nonpolar, halides are electron-withdrawing, and so forth. Ab initio calculations spend much of their time in re-calculating the transferable characteristics of functional groups. Semiempirical approximations replace the ab initio Hamiltonian with a simpler model Hamiltonian, which contains parameters that capture the transferable characteristics of functional groups. Examples of these parameters include force constants in molecular mechanics [19] or Hamiltonian matrix elements in semiempirical quantum-mechanical approximations [20, 21].

One of the benefits of $\mathcal{O}(\mathrm{N})$ methods is their controllability. $\mathcal{O}(\mathrm{N})$ approximations yield well-defined changes in accuracy and computational effort. The decision to use an $\mathcal{O}(\mathrm{N})$ approximation can be made a priori based on the size of the system of interest [3] .

Unfortunately, semiempirical methods usually involve a significant trade-off between com- 
putational effort and accuracy. Semiempirical methods are much less accurate than ab initio methods for many systems. This has led to the widespread use of "hybrid" QM/MM methods, a nearsightedness-based tradeoff between ab initio accuracy and semiempirical speed [22, 23]. Our goal is to systematically improve semiempirical theory.

Most existing semiempirical methods are based on models that were designed to be parametrized to experimental data. Though many semiempirical methods are now parametrized using $a b$ initio results (e.g Refs. [24, 25, 26, 27]), we believe that the existing methods may not take full advantage of the possibilities inherent in $a b$ initio parametrization. $A b$ initio calculations on small molecules can give orders of magnitude more parametrization data than can be readily obtained from experiment. They also yield information that is more directly relevant to a semiempirical model's parameters.

Nearsightedness and molecular similarity suggest that we can model large systems as the sum of contributions from different functional groups. This implies that a sufficiently rich data set of a functional group in small molecules will contain all information needed to describe the functional group in molecules of arbitrary size. Our overall approach is to generate rich data sets on the behavior of functional groups by doing a large number of highly accurate $a b$ initio calculations on the group in a set of small-molecule environments. This paper investigates whether a semiempirical model parametrized to this sort of smallmolecule data can give ab initio accuracy for larger molecules.

This approach is fairly general. It requires only that the semiempirical model can describe a system as a sum of subsystem contributions. For example, a semiempirical model that predicts the amplitudes of delocalized wavefunctions would not be compatible with this approach.

The current work details our first implementation of this approach: a semiempirical subsystem-based treatment of electron correlation. We model the system in terms of its oneand two-electron density matrices in an atomic orbital basis set (Sec. ПA). Subsystem twoelectron density matrices are combined to model the two-electron density matrix of the entire system. This model was chosen because it treats an important problem in contemporary electron structure theory (electron correlation), and because the predicted outputs (twoelectron density matrices) are much easier to obtain from $a b$ initio calculation than from experiment. 


\section{METHODS}

\section{A. Semiempirical model for electron correlation}

Our semiempirical model treats electron correlation by predicting subsystem two-electron density matrices as a functional of subsystem one-electron density matrices. A system's one- and two-electron density matrices ${ }^{1} D,{ }^{2} D$ are obtained from its normalized $N$-electron wavefunction $|\Phi\rangle$ as

$$
\begin{gathered}
{ }^{1} D(a, b)=\left\langle\Phi\left|a_{a}^{\dagger} a_{b}\right| \Phi\right\rangle \\
{ }^{2} D(a c, b d)=1 / 2\left\langle\Phi\left|a_{a}^{\dagger} a_{c}^{\dagger} a_{b} a_{d}\right| \Phi\right\rangle
\end{gathered}
$$

in second quantization with one-electron basis functions $\left\{\left|\phi_{a}\right\rangle\right\}$. For an N-electron system, the trace of ${ }^{1} D$ equals $\mathrm{N}$ and the trace of ${ }^{2} D$ equals the number of unique electron pairs, $1 / 2 N(N-1)$. The electron-electron interaction energy of a system $\left(E_{2}\right)$ is obtained as the trace over the product of the two-electron integrals and the two-electron density matrix

$$
\begin{aligned}
E_{2} & =\sum_{a b c d}\langle a c \mid b d\rangle^{2} D(a c, b d) \\
\langle a c \mid b d\rangle & \equiv \int d \mathbf{r}_{1} d \mathbf{r}_{2} \phi_{a}^{*}\left(\mathbf{r}_{1}\right) \phi_{c}^{*}\left(\mathbf{r}_{2}\right) \frac{1}{\left|\mathbf{r}_{2}-\mathbf{r}_{1}\right|} \phi_{b}\left(\mathbf{r}_{1}\right) \phi_{d}\left(\mathbf{r}_{2}\right)
\end{aligned}
$$

The electron density in real space is the diagonal of the one-electron density matrix: ${ }^{1} D(\mathbf{r}) \equiv$ $\left\langle\Phi\left|a_{\mathbf{r}}^{\dagger} a_{\mathbf{r}}\right| \Phi\right\rangle[28] .{ }^{1} D$ and ${ }^{2} D$ provide a complete description of a system whose Hamiltonian contains only one- and two-body interactions [29].

The two-electron density matrix ${ }^{2} D$ obtained from $|\Phi\rangle$ can be expressed as a cumulant expansion [30, 31]

$$
\begin{aligned}
{ }^{2} D(a c, b d) & =1 / 2{ }^{1} D(a, b){ }^{1} D(c, d) \\
& -1 / 2{ }^{1} D(a, d){ }^{1} D(b, c) \\
& +{ }^{2} \Delta(a c, b d)
\end{aligned}
$$

where the three terms on the right-hand side of Eq. 4 are denoted Coulomb, exchange, and correlation contributions to ${ }^{2} D$. The connected pair density matrix ${ }^{2} \Delta$ is that part of ${ }^{2} D$ that cannot be written as a simple function of ${ }^{1} D$. The Coulomb and exchange contributions to ${ }^{2} \mathrm{D}$ in Eq. 4 are well-approximated at the Hartree and Hartree-Fock levels of theory, respectively. However, accurate ab initio treatment of the connected pair density matrix ${ }^{2} \Delta$ requires expensive high-level methods. 
Density functional theory (DFT) is a formally exact method for treating a system of interacting electrons exclusively in terms of its one-electron density [29, 32, 33]. The electron-electron interaction energy of Eq. 3 is treated as the sum of a Coulomb term and an exchange-correlation correction $E_{X C}$, such that the electrons move in a potential that is corrected by the exchange-correlation potential $v_{X C}(\mathbf{r})=\delta\left(E_{X C}\right) / \delta\left({ }^{1} D(\mathbf{r})\right)$. DFT is implemented by approximating $v_{X C}$ as a functional of electron density: $v_{X C}=v_{X C}\left[{ }^{1} D(\mathbf{r})\right]$. (In Kohn-Sham DFT, the kinetic energy is decomposed into the kinetic energy of the Kohn-Sham orbitals plus a density-dependent correction, which is incorporated into $v_{X C}$ via e.g. adiabatic connection [29]. Our $v_{\text {corr }}$ functionals (Eq. 9) do not include a kinetic energy correction.) Following Eq. 3] and Eq. 4. $E_{X C}$ may be obtained as the trace over the exchange and correlation contributions to the two-electron density matrix: $E_{X C}=\sum\langle a c \mid b d\rangle\left(-1 / 2{ }^{1} D(a, d){ }^{1} D(b, c)+{ }^{2} \Delta(a c, b d)\right)$. Thus, the correlation component $v_{\text {corr }}\left[{ }^{1} D\right]$ of a system's exchange-correlation functional can be obtained from the first derivative of a functional that predicts a system's connected pair density matrix ${ }^{2} \Delta$ as a function of its electron density matrix ${ }^{1} D$ :

$$
\begin{array}{r}
v_{c o r r}\left[{ }^{1} D\right]\left(a^{\prime}, b^{\prime}\right)=\sum_{a b c d}\langle a c \mid b d\rangle \\
\times \delta\left({ }^{2} \Delta\left[{ }^{1} D\right](a c, b d)\right) / \delta\left({ }^{1} D\left(a^{\prime}, b^{\prime}\right)\right)
\end{array}
$$

Explicit treatments of $v_{X C}\left[{ }^{1} D\right]$ in terms of the two-electron density include various analyses of the real-space exchange-correlation hole [34, 35, 36].

In this work, we define the correlation energy $E_{\text {corr }}$ as the expectation value of the connected pair density matrix: $E_{c o r r}=\sum\langle a c \mid b d\rangle^{2} \Delta(a c, b d)$. Correlation energy can also be defined as the difference in energies predicted by configuration-interaction and Hartree-Fock calculations: $E_{c o r r}=E_{C I}-E_{H F}$. The latter definition includes the effects of ${ }^{1} D$ relaxation, e.g. the expectation value of ${ }^{1} D_{C I}-{ }^{1} D_{H F}$. In contrast, the former definition yields the correlation energy corresponding to a single choice of ${ }^{1} D$, and is therefore consistent with the definition of $E_{\text {corr }}$ used in DFT and MP2 calculations.

Both ${ }^{1} D$ and ${ }^{2} \Delta$ can be treated using the nearsightedness approximation. Several nearsightedness-based treatments of ${ }^{1} \mathrm{D}$ exist, including divide-and-conquer methods that partition ${ }^{1} D$ into subsystem contributions as in Fig. 1 [3, 7, 37, 38, 39, 40]. Nearsightednessbased treatments of ${ }^{2} \Delta$ include the $\mathcal{O}(\mathrm{N})$ treatments of electron correlation cited previously 10, 11, 12, 13, 14, 15, 16, 17, 18]. We recently developed the "localized reduced 
density matrix" (LRDM) method [41], a divide-and-conquer style treatment of ${ }^{2} \Delta$. LRDM assembles a large system's atomic-orbital-basis ${ }^{2} \Delta$ from the results of $a b$ initio calculations on overlapping subsystems. Like other divide-and-conquer methods, LRDM is non-variational.

In the current work, we use LRDM as a framework for semiempirical subsystem-based approximations for DFT correlation functionals $v_{\text {corr }}\left[{ }^{1} D\right]$. We generate semiempirical functionals that predict the matrix elements of a subsystem's ${ }^{2} \Delta$ as a function of the subsystem electron density matrix ${ }^{1} D:{ }^{2} \Delta\left[{ }^{1} D\right]$ (see Eq. [7] and Eq. 9 below). An approximate ${ }^{2} \Delta$ for a large system is obtained by combining subsystem ${ }^{2} \Delta\left[{ }^{1} D\right]$ predictions using LRDM. Our results indicate that basis-set ${ }^{2} \Delta\left[{ }^{1} D\right]$ functionals can provide good results for multiple subsystem geometries (Sec. III).

LRDM can treat long-range correlations (dispersion interactions) by doing ab initio calculations that include correlation in two disjoint regions of a molecule [41]. In the current work, we do not model these long-range interactions. Therefore, our subsystem-based $v_{\text {corr }}\left[{ }^{1} D\right]$ functionals, like standard DFT $v_{\text {corr }}$ functionals [4, 43], cannot treat dispersion interactions.

Our subsystem-based $v_{\text {corr }}\left[{ }^{1} D\right]$ functionals are very different than the standard DFT functionals derived from the homogeneous electron gas [29, 33, 44, 45]. Other groups have developed $v_{X C}\left[{ }^{1} D\right]$ functionals that are semiempirical [46, 47], subsystem-based [48, 49] or fitted to high-accuracy ab initio data 50, 51, 52], but to our knowledge the current method is unique in combining semiempirical methods with a nearsighted, molecular-similarity-based treatment of ${ }^{2} \Delta$.

\section{B. Parametrization method}

Our approach is to develop semiempirical models that are parametrized using rich data sets of small-molecule $a b$ initio calculations. These rich data sets allow us to use data mining methods in the parametrization stage. "Data mining" refers to computational methods for analyzing large data sets and automatically extracting previously unknown dependencies between the data 53 . Other data-mining treatments of electron correlation include a neuralnetwork exchange-correlation potential fitted to data from many molecules [50], and a model for the correlation energy between pairs of widely separated, localized electrons [54].

Data mining methods can determine two types of relationships between data. The first 
is the system's dimensionality: which input and output descriptors are most important for describing the data set. (Here, "descriptor" is a generic term for a type of data used by a model. For example, the input and output descriptors of our ${ }^{2} \Delta\left[{ }^{1} D\right]$ functionals are ${ }^{1} D(a, b)$ and ${ }^{2} \Delta(a c, b d)$ matrix elements.) The second type of relationship that can be determined by data mining is the functional form of the [input descriptor] $\rightarrow$ [output descriptor] relation. In the current work, we assume a quadratic input-output relation and focus on dimensional reduction.

A flowchart of the data-mining process for a functional group is as follows.

1. Choose an initial set of input and output descriptors, and a fit function to relate them. As discussed in Sec. [I subsystem-based models require input and output descriptors that describe electronic structure in terms of local information (e.g. electron densities). Since the models are meant for use within semiempirical models, the input descriptors should be obtainable from a simple approximate Hamiltonian (e.g. the DFT Hamiltonian). The fit function can be anything from a polynomial fit to a neural network.

2. Generate an initial data set of $a b$ initio calculations on the functional group in various small molecules and environments. Extract the values of all input and output descriptors for each point in the data set.

3. Split the data set into training and testing subsets.

4. Reduce the dimensionality of the data set, by using (for example) principal component analysis to determine a few combinations of descriptors that capture most of the variation in the data set. The model will be parametrized on this dimensionallyreduced input and output data.

5. Parametrize the model using the training subset of the small-molecule data.

6. Test the model on the testing subset of the small-molecule data, and on larger molecules.

As stated above, our initial focus is on the dimensional reduction of ${ }^{1} D$ and ${ }^{2} \Delta$ (step 4). For a system with $M$ basis functions, ${ }^{1} D$ and ${ }^{2} \Delta$ have $1 / 2\left(M^{2}+M\right)-1$ and $1 / 8\left(M^{4}+\right.$ $\left.2 M^{3}+5 M^{2}+4 M\right)-1$ degrees of freedom, respectively. Without dimensional reduction, even 
a small subsystem (e.g. $\mathrm{M}=\mathcal{O}(10)$ ) has far too many output degrees of freedom for a ${ }^{2} \Delta\left[{ }^{1} D\right]$ functional to be useful. We use principal component analysis (PCA) to decompose ${ }^{1} D$ and ${ }^{2} \Delta$ into a set of importance-weighted basis functions. To illustrate, PCA on a set of subsystem electron density matrices $\left\{{ }^{1} D_{\sigma}\right\}$ describes each density matrix as

$$
{ }^{1} D_{\sigma}(a, b)={ }^{1} D_{\text {avg }}(a, b)+\sum_{i} c_{\sigma i}{ }^{1} D_{i}(a, b)
$$

where ${ }^{1} D_{\text {avg }}$ is the average electron density matrix, ${ }^{1} D_{i}$ are the principal components, and the standard deviation of the expansion coefficients $c_{\sigma i}$, evaluated across the data points $\sigma$, decreases with increasing $i[53]$.

In this work, we use a quadratic function to predict the first few (most important) ${ }^{2} \Delta$ components from the first few ${ }^{1} D$ components. A quadratic function is the lowest-order polynomial of ${ }^{2} \Delta\left[{ }^{1} D\right]$ for which the associated correlation-energy functional $v_{\text {corr }}\left[{ }^{1} D\right]$ (Eq. 5 ) is not a constant. The ${ }^{2} \Delta\left[{ }^{1} D\right]$ functionals fit the first $C_{2}$ principal components of ${ }^{2} \Delta$ as a function of the first $C_{1}$ components of ${ }^{1} D$ such that

$$
\begin{array}{r}
{ }^{2} \Delta\left[{ }^{1} D\right](a c, b d)={ }^{2} \Delta_{a v g}(a c, b d)+\sum_{j}^{C_{2}}\left\{{ }^{2} \Delta_{j}(a c, b d)\right. \\
\left.\times\left(\alpha_{j}+\sum_{i}^{C_{1}}\left(\gamma_{i j}\left({ }^{1} D \mid{ }^{1} D_{i}\right)+\sigma_{i j}\left({ }^{1} D \mid{ }^{1} D_{i}\right)^{2}\right)\right)\right\}
\end{array}
$$

where ${ }^{2} \Delta_{j}$ are the principal components of ${ }^{2} \Delta,\left(\left.{ }^{1} D\right|^{1} D_{i}\right)$ is the projection of the argument one-electron density matrix ${ }^{1} D$ onto the $i$ th principal component

$$
\left({ }^{1} D \mid{ }^{1} D_{i}\right) \equiv \sum_{a b}\left({ }^{1} D(a, b)-{ }^{1} D_{\text {avg }}(a, b)\right){ }^{1} D_{i}(a, b)
$$

and $\left\{\alpha_{j}, \gamma_{i j}, \sigma_{i j}\right\}$ are fitted parameters. Each component of the two-electron density matrix is fit independently of the others. The subsystem DFT correlation energy operator $v_{\text {corr }}\left[{ }^{1} D\right]$ is obtained from ${ }^{2} \Delta\left[{ }^{1} D\right]$, following Eq. 5 , as

$$
\begin{array}{r}
\left(v_{\text {corr }}\left[{ }^{1} D\right]\right)\left(a^{\prime}, b^{\prime}\right)=\sum_{a b c d}\langle a c \mid b d\rangle \sum_{j}^{C_{2}}\left\{{ }^{2} \Delta_{j}(a c, b d)\right. \\
\left.\times \sum_{i}^{C_{1}}{ }^{1} D_{i}\left(a^{\prime}, b^{\prime}\right)\left(\gamma_{i j}+2 \sigma_{i j}\left({ }^{1} D \mid{ }^{1} D_{i}\right)\right)\right\}
\end{array}
$$

The $v_{\text {corr }}\left[{ }^{1} D\right]$ functional of a large system is obtained by overlaying subsystem contributions as in LRDM (Fig. (1). The degree of dimensional reduction can be seen by comparing the 
number of fitted components $C_{1}$ and $C_{2}$ to the total number of degrees of freedom in ${ }^{1} D$ and ${ }^{2} \Delta$.

The approach discussed here can be used to construct many different kinds of semiempirical model based on the choice of input and output descriptors. For example, we have begun work on a semiempirical model of core polarization in effective core potentials [55]. Here, the input descriptors are the valence electron density matrix and one-electron Hamiltonian, and the output descriptors are the core electron density matrices. This work will be discussed in a future publication.

The principal computational challenge of this approach is the steep scaling of the amount of training set data required. The ${ }^{2} \Delta\left[{ }^{1} D\right]$ functional of an M-orbital subsystem will have $\mathrm{I}=\mathcal{O}\left(\mathrm{M}^{2}\right){ }^{1} D_{i}$ input components [56]. In general, a function with I input components

must be parametrized using $\mathcal{O}\left(e^{\mathrm{I}}\right)$ data points [53]. Because of this, we have focused our initial work on proof-of-concept treatments for small model systems in minimal basis sets. Successful application of our method to these systems will justify expanding our focus to larger, chemically-interesting systems and larger basis sets.

\section{Error Decomposition}

Our ${ }^{2} \Delta\left[{ }^{1} D\right]$ functionals contain three distinct approximations. The first approximation is nearsightedness: the connected pair density matrix ${ }^{2} \Delta$ is assumed to be well-described by a decomposition into overlapping subsystems. The second approximation is that each subsystem ${ }^{2} \Delta$ is assumed to be well-described by a relatively small number of principal components $\left(C_{2}\right.$ in Eq. (7). The third approximation is that each subsystem ${ }^{2} \Delta$ component is assumed to be well-described by the ${ }^{1} D$ functional in Eq. 7 .

We can isolate the effects of each of these assumptions using three kinds of approximate connected pair density matrix (see Table 【). The first approximate connected pair density matrix is the "exact subsystem" connected pair density matrix: ${ }^{2} \Delta_{x s u b}$. This is obtained by projecting the correct ${ }^{2} \Delta$ onto the overlapping subsystems, and setting to zero all matrix elements that are not contained within a subsystem. The second is the principal component reduction connected pair density matrix: ${ }^{2} \Delta_{P C A}$. This is obtained by projecting the subsystem blocks of ${ }^{2} \Delta_{x s u b}$ onto the ${ }^{2} \Delta$ components that are fitted by the subsystem ${ }^{2} \Delta\left[{ }^{1} D\right]$ functionals $[57]$. The third is the connected pair density matrix obtained using the subsys- 
tem ${ }^{2} \Delta\left[{ }^{1} D\right]$ functional and the correct one-electron density matrix: ${ }^{2} \Delta\left[{ }^{1} D_{\text {exact }}\right]$. Table I] summarizes the approximations used in generating these density matrices.

\section{RESULTS}

The remainder of this paper details demonstrations of our semiempirical subsystem-based ${ }^{2} \Delta\left[{ }^{1} D\right]$ functionals. We begin by demonstrating ${ }^{2} \Delta\left[{ }^{1} D\right]$ functionals for a linear dimerized chain of minimal-basis hydrogen atoms $(\mathrm{H}-\mathrm{H})_{5}$, since the functional predictions can be readily compared to full-CI. Then, we demonstrate that a ${ }^{2} \Delta\left[{ }^{1} D\right]$ functional for the aldehyde group, parametrized to data from a set of small HOC-R molecules, can extrapolate to $\mathrm{R}$ groups outside of the training set. All ab initio calculations were performed using a modified version of the GAMESS electronic structure program [58].

\section{A. $(\mathbf{H}-\mathbf{H})_{2}$ and $(\mathbf{H}-\mathbf{H})_{5}$ systems}

The first system is linear minimal-basis $(\mathrm{H}-\mathrm{H})_{5}$. This system is treated as a sum of four overlapping $(\mathrm{H}-\mathrm{H})_{2}$ subsystems. We model its correlation energy by parametrizing a $(\mathrm{H}-\mathrm{H})_{2}$ ${ }^{2} \Delta\left[{ }^{1} D\right]$ functional to data on isolated $(\mathrm{H}-\mathrm{H})_{2}$ molecules, and combining the $(\mathrm{H}-\mathrm{H})_{2}{ }^{2} \Delta\left[{ }^{1} D\right]$ predictions using LRDM [59]. The functionals are parametrized to, and tested on, full-CI ab initio calculations.

We generated data for both variable- and fixed-geometry molecules, yielding the four data sets in Table II] Each molecule was electrostatically perturbed by randomly placing

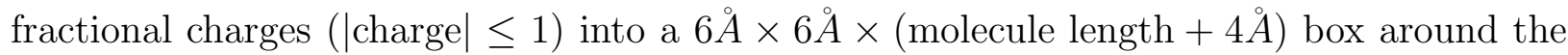
molecule, with a minimum point charge - atom separation of $1.2 \AA$. Variable geometry systems had each bond length set randomly within the ranges in Table II

The $(\mathrm{H}-\mathrm{H})_{2}{ }^{2} \Delta\left[{ }^{1} \mathrm{D}\right]$ functionals were parametrized using half of the $(\mathrm{H}-\mathrm{H})_{2}$ data as a training set (see item 3 in the flowchart of Sec. [IB). Separate functionals were parametrized for the variable- and fixed-geometry systems. The numbers of principal components included in the ${ }^{2} \Delta\left[{ }^{1} D\right]$ functionals $\left(C_{1}\right.$ and $C_{2}$ in Table II) were selected to give good results for both ${ }^{2} \Delta\left[{ }^{1} D_{\text {exact }}\right]$ and ${ }^{2} \Delta\left[{ }^{1} D_{D F T}\right]$ (see below). The principal component analyses were a significant dimensional reduction, as the ${ }^{1} D$ and ${ }^{2} \Delta$ of $(\mathrm{H}-\mathrm{H})_{2}$ contain 9 and 59 degrees of freedom, respectively. 


\section{B. Modeling (H-H $)_{2}$ using $(\mathbf{H}-\mathbf{H})_{2}{ }^{2} \Delta\left[{ }^{1} D\right]$ functionals}

The first test of the $(\mathrm{H}-\mathrm{H})_{2}{ }^{2} \Delta\left[{ }^{1} \mathrm{D}\right]$ functionals is how well they can predict the $(\mathrm{H}-\mathrm{H})_{2}$ ${ }^{2} \Delta$ given the correct full-CI electron density matrix ${ }^{1} D_{\text {exact }}$. Fig. 2 plots predicted vs. real $E_{\text {corr }}$ for the $(\mathrm{H}-\mathrm{H})_{2}$ systems. Table III presents $\left|\delta E_{\text {corr }}\right|$ errors averaged over the trainingand testing-set data. Here, $\left|\delta E_{\text {corr }}\right|$ is the absolute error in the predicted correlation energy $E_{c o r r}=\sum_{a b c d}\langle a c \mid b d\rangle{ }^{2} \Delta(a c, b d)$ (see Eq. 3). The ${ }^{2} \Delta\left[{ }^{1} D\right] E_{\text {corr }}$ predictions are compared to MP2.

The results are quite encouraging. The ${ }^{2} \Delta\left[{ }^{1} D\right]$ functionals are better than MP2 at predicting the average value of the correlation energy: the mean absolute errors in $E_{\text {corr }}$ from MP2 are 40 and 150 times as large as the error for the ${ }^{2} \Delta\left[{ }^{1} D\right]$ functionals (variable and fixed geometry, respectively). ${ }^{2} \Delta\left[{ }^{1} D\right]$ functionals are also better than MP2 at predicting the variation of the correlation energy across the data set. This can be seen in Fig. 2 2 , the slope of the predicted vs. real $E_{\text {corr }}$ values is very small for the MP2 predictions but close to 1 for the ${ }^{2} \Delta\left[{ }^{1} D\right]$ functionals. Despite its low scatter, MP2 does not capture either the value or the variation in the correlation energy.

The scatter in the ${ }^{2} \Delta\left[{ }^{1} D\right]$ predictions for the fixed-geometry system can be reduced by parametrizing a ${ }^{2} \Delta\left[{ }^{1} D\right]$ functional that uses more principal components. We parametrized a ${ }^{2} \Delta\left[{ }^{1} D\right]$ functional for fixed-geometry $(\mathrm{H}-\mathrm{H})_{2}$ that includes seven ${ }^{1} D$ and eight ${ }^{2} \Delta$ principal components $\left(C_{1}=7, C_{2}=8\right.$ in Eq. 7). This functional gives an $\mathrm{R}^{2}$ between real and predicted $E_{\text {corr }}$ of 0.990 , comparable to the 0.991 value for MP2 and better than the 0.890 value in Fig. 22 For this functional, the average (standard deviation) ${ }^{2} \Delta\left[{ }^{1} D_{\text {exact }}\right]\left|\delta E_{\text {corr }}\right|$ values are $0.11(0.29) \mathrm{mH}$ for the testing-set data.

A comparison of the ${ }^{2} \Delta_{P C A}$ and ${ }^{2} \Delta\left[{ }^{1} D_{\text {exact }}\right]$ errors in Table III] shows that most of the error in the variable-geometry system is due to dimensional reduction of ${ }^{2} \Delta$, as the ${ }^{2} \Delta_{P C A}$ errors are almost as large as the corresponding ${ }^{2} \Delta\left[{ }^{1} D_{\text {exact }}\right]$ errors. In contrast, the error in the fixed-geometry system is more evenly partitioned between dimensional reduction of ${ }^{2} \Delta$ and prediction of ${ }^{2} \Delta$ from ${ }^{1} D$.

The training- and testing-set errors are reasonably close to each other, indicating that the functionals are not over-fitted. We tested a second measure of the predicted ${ }^{2} \Delta$, the sum of absolute errors in the predicted ${ }^{2} \Delta$ matrix elements. These errors were fairly well-correlated with the $\left|\delta E_{\text {corr }}\right|$ errors presented above (data not shown). 
The results in Table $\llbracket$ and Fig. [2 show that the constant ${ }^{2} \Delta$ returned by ${ }^{2} \Delta\left[{ }^{1} D_{\text {avg }}\right]$ is a surprisingly good approximation for the variable-geometry systems. This is encouraging, as it suggests that even the most primitive ${ }^{2} \Delta\left[{ }^{1} D\right]$ functional (e.g. a constant ${ }^{2} \Delta$ ) can work reasonably well for multiple subsystem geometries. Our ${ }^{2} \Delta\left[{ }^{1} D\right]$ functionals all improve upon this primitive functional, as all ${ }^{2} \Delta\left[{ }^{1} D_{\text {exact }}\right]$ errors are lower than the corresponding ${ }^{2} \Delta\left[{ }^{1} D_{\text {avg }}\right]$ errors. As expected, ${ }^{2} \Delta\left[{ }^{1} D_{\text {avg }}\right]$ predicts a constant correlation energy for the fixed-geometry systems (Fig. 2).

\section{Modeling $(\mathbf{H}-\mathbf{H})_{5}$ using $(\mathbf{H}-\mathbf{H})_{2}{ }^{2} \Delta\left[{ }^{1} D\right]$ functionals}

The results in Fig. 2 and Table III demonstrate that the $(\mathrm{H}-\mathrm{H})_{2}{ }^{2} \Delta\left[{ }^{1} D\right]$ functionals give good ${ }^{2} \Delta$ predictions for $(\mathrm{H}-\mathrm{H})_{2}$. Given this, we investigate whether four copies of an $(\mathrm{H}-\mathrm{H})_{2}{ }^{2} \Delta\left[{ }^{1} D\right]$ functional, combined using LRDM, will suffice to describe correlation effects in $(\mathrm{H}-\mathrm{H})_{5}$. Using the $(\mathrm{H}-\mathrm{H})_{2}{ }^{2} \Delta\left[{ }^{1} \mathrm{D}\right]$ functional on $(\mathrm{H}-\mathrm{H})_{5}$ tests whether the fundamental assumptions of nearsightedness and molecular similarity, and our implementation of these approximations, are correct for the $(\mathrm{H}-\mathrm{H})_{5}$ model system. Fig. [3 and Table $\mathbb{1 V}$ present data for $(\mathrm{H}-\mathrm{H})_{5}$ systems, using the notation of Fig. 2 and Table III.

The $(\mathrm{H}-\mathrm{H})_{5}$ results are also encouraging. Four copies of an $(\mathrm{H}-\mathrm{H})_{2}{ }^{2} \Delta\left[{ }^{1} D\right]$ functional, combined via LRDM, are better than MP2 at describing the mean and variation of $E_{\text {corr }}$ for the $(\mathrm{H}-\mathrm{H})_{5}$ system. The mean absolute $E_{\text {corr }}$ errors for MP2 are 60 and 90 times the values for ${ }^{2} \Delta\left[{ }^{1} D\right]$ functionals (variable and fixed geometry, respectively). Fig. 3 shows that our method does better than MP2 at capturing the variation in $E_{\text {corr }}$ across the data set, with a predicted vs. real $E_{\text {corr }}$ whose slope is very small for MP2 but near one for our method.

For the variable-geometry systems, the ${ }^{2} \Delta\left[{ }^{1} D\right]$ functionals describe the $(\mathrm{H}-\mathrm{H})_{5}$ data to about the same level of accuracy (per atom) as the $(\mathrm{H}-\mathrm{H})_{2}$ data. The average $(\mathrm{H}-\mathrm{H})_{5}\left|\delta E_{\text {corr }}\right|$ are about $10 / 4=2.5$ times as large as the corresponding $(\mathrm{H}-\mathrm{H})_{2}$ values. For example, the average ${ }^{2} \Delta\left[{ }^{1} D_{\text {exact }}\right]$ error is $1.91 \mathrm{mH}$ for variable-geometry $(\mathrm{H}-\mathrm{H})_{2}$ and $3.43 \mathrm{mH}$ for variablegeometry $(\mathrm{H}-\mathrm{H})_{5}$.

For the fixed-geometry systems, the ${ }^{2} \Delta\left[{ }^{1} D\right]$ functionals do not describe the $(\mathrm{H}-\mathrm{H})_{5}$ data to the same level of accuracy as the $(\mathrm{H}-\mathrm{H})_{2}$ data: the average $(\mathrm{H}-\mathrm{H})_{5}\left|\delta E_{\text {corr }}\right|$ are about five times as large as the corresponding $(\mathrm{H}-\mathrm{H})_{2}$ values. This error is not due to the subsystem decomposition: the average $\left|\delta E_{\text {corr }}\right|$ of ${ }^{2} \Delta_{\text {xsub }}$ is only $0.02 \mathrm{mH}$ (Table IV). We suggest that 
the long-range order in the fixed-geometry $(\mathrm{H}-\mathrm{H})_{5}$ leads to an intrinsic difference between the environments experienced by an isolated $(\mathrm{H}-\mathrm{H})_{2}$ vs. an $(\mathrm{H}-\mathrm{H})_{2}$ embedded in $(\mathrm{H}-\mathrm{H})_{5}$. Better $(\mathrm{H}-\mathrm{H})_{2}{ }^{2} \Delta\left[{ }^{1} \mathrm{D}\right]$ functionals for the fixed-geometry systems could perhaps be generated by using cyclic boundary conditions in the $(\mathrm{H}-\mathrm{H})_{2}$ data. Evidence for this conclusion is discussed in the Supporting Information.

The predictions of a semiempirical model should not depend on the choice of training set used to parametrize the model. We parametrized $(\mathrm{H}-\mathrm{H})_{2}{ }^{2} \Delta\left[{ }^{1} D\right]$ functionals using multiple choices of training set. Results are discussed in the Supporting Information. As expected, the functionals have only a weak dependence on training set choice.

\section{DFT calculations with ${ }^{2} \Delta\left[{ }^{1} D\right]$ functionals}

The above results test the ${ }^{2} \Delta\left[{ }^{1} D\right]$ functional's predictions given the correct electron density matrix ${ }^{1} D_{\text {exact }}$. However, the functionals are intended for use in density functional theory (Sec. 【A ) where ${ }^{1} D_{\text {exact }}$ is not known in advance. We have implemented two methods for using the functionals. The first is DFT with exact exchange and the ${ }^{2} \Delta\left[{ }^{1} D\right]$ correlation functional of Eq. 9, referred to as ${ }^{2} \Delta\left[{ }^{1} D_{D F T}\right]$. The second method, like MP2, is a one-step post-Hartree-Fock prediction of $E_{\text {corr }}$. Here, the correct electron density matrix ${ }^{1} D_{\text {exact }}$ is approximated as the Hartree-Fock electron density matrix ${ }^{1} D_{H F}$, and the correlation energy is obtained non-self-consistently from ${ }^{2} \Delta\left[{ }^{1} D_{H F}\right]$.

Table $\mathrm{V}$ presents $\left|\delta E_{\text {corr }}\right|$ values for ${ }^{2} \Delta\left[{ }^{1} D_{D F T}\right]$ and ${ }^{2} \Delta\left[{ }^{1} D_{H F}\right]$ on the fixed- and variablegeometry $(\mathrm{H}-\mathrm{H})_{2}$ and $(\mathrm{H}-\mathrm{H})_{5}$ systems, for a single choice of training set. Predicted vs. real $E_{\text {corr }}$ for the $(\mathrm{H}-\mathrm{H})_{5}$ systems are plotted in Fig. 4.

The ${ }^{2} \Delta\left[{ }^{1} D_{D F T}\right]$ calculations do a fairly good job of predicting the average and variation in ${ }^{2} \Delta$ and $E_{\text {corr }}$ : the average and standard deviations in $\left|\delta E_{\text {corr }}\right|$ are much better than MP2, and the standard deviations in $\left|\delta E_{\text {corr }}\right|$ are generally smaller than the primitive ${ }^{2} \Delta\left[{ }^{1} D_{\text {avg }}\right]$ functional (see Tables III and [V]). These results are encouraging, given that our $v_{\text {corr }}\left[{ }^{1} D\right]$ functional is a simple linear function of ${ }^{1} \mathrm{D}$ (see Eq. 9). The results from the $(\mathrm{H}-\mathrm{H})_{5}$ systems are especially encouraging: four identical, overlapping $(\mathrm{H}-\mathrm{H})_{2} v_{\text {corr }}\left[{ }^{1} \mathrm{D}\right]$ functionals give a reasonable prediction for the $v_{\text {corr }}\left[{ }^{1} \mathrm{D}\right]$ of $(\mathrm{H}-\mathrm{H})_{5}$. Fig. 4 shows that the relatively large average errors in the ${ }^{2} \Delta\left[{ }^{1} D_{D F T}\right]\left|\delta E_{\text {corr }}\right|$ are mostly systematic error. Better ${ }^{2} \Delta\left[{ }^{1} D_{D F T}\right]$ results could perhaps be generated using a more sophisticated (nonlinear) function (see flowchart, 
Sec. [IB). The errors in the non-self-consistent ${ }^{2} \Delta\left[{ }^{1} D_{H F}\right]$ calculations are somewhat higher than the self-consistent ${ }^{2} \Delta\left[{ }^{1} D_{D F T}\right]$ calculations. This is reasonable, especially given that ${ }^{1} D_{H F}$ is not necessarily a good approximation for ${ }^{1} D_{\text {exact }}$.

When ${ }^{2} \Delta\left[{ }^{1} D\right]$ functionals are combined with exact exchange, the ${ }^{2} \Delta\left[{ }^{1} D_{H F}\right]$ and ${ }^{2} \Delta\left[{ }^{1} D_{D F T}\right]$ calculations give a fairly large, systematic under-estimate of the total energy. This is partly due to a difference between the ${ }^{1} D$ obtained using full-CI and HF theory on minimal-basis $(\mathrm{H}-\mathrm{H})_{5}$. For a closed-shell N-electron system, the combined trace of the exchange and correlation parts of ${ }^{2} D$ (Eq. 4) equals $-1 / 2 N$ [29]. Full-CI calculations on $(\mathrm{H}-\mathrm{H})_{5}$ give ${ }^{2} \Delta$ with a trace $<0$ and an exact exchange pair density matrix ${ }^{2} D_{X}(a c, b d)=-1 / 2{ }^{1} D(a, d){ }^{1} D(b, c)$ with a trace less than $-1 / 2 N$. Thus, all of the $(\mathrm{H}-\mathrm{H})_{2}{ }^{2} \Delta\left[{ }^{1} D\right]$ functionals return a ${ }^{2} \Delta$ with a negative trace. However, ab initio methods that return a single-determinant wavefunction (e.g. HF or KS-DFT theory) give a closed-shell ${ }^{2} D_{X}$ with a trace equal to $-1 / 2 N$. Thus, for example, the approximate two-electron density matrix returned by our non-self-consistent method ${ }^{2} D(a c, b d)=$ $1 / 2{ }^{1} D_{H F}(a, c){ }^{1} D_{H F}(b, d)-1 / 2{ }^{1} D_{H F}(a, d){ }^{1} D_{H F}(b, c)+{ }^{2} \Delta\left[{ }^{1} D_{H F}\right](a c, b d)$ will always have a trace less than the correct value $(1 / 2 N(N-1)$, see Sec. [A $)$. This leads to a systematic under-estimate of the number of electron pairs in the system and the electron-electron interaction energy. One way to correct this is by renormalizing the exact-exchange ${ }^{2} D_{X}$ obtained from ${ }^{1} D_{H F}$ or ${ }^{1} D_{D F T}$, such that the final predicted ${ }^{2} D$ has the correct trace. This is analogous to the use of a fraction of exact exchange in "hybrid" DFT functionals such

as B3LYP [46]. This significantly improves the total energies: for example, the average (standard deviation) total energy error for variable-geometry $(\mathrm{H}-\mathrm{H})_{5}$ is $114.71(13.50) \mathrm{mH}$ for uncorrected Hartree-Fock calculations and -71.52 (7.26) $\mathrm{mH}$ and -160.88 (19.67) $\mathrm{mH}$ for ${ }^{2} \Delta\left[{ }^{1} D_{H F}\right]$ with and without renormalization of ${ }^{2} D_{X}$.

\section{E. Substituted aldehydes}

The assumption of molecular similarity implies that a ${ }^{2} \Delta\left[{ }^{1} D\right]$ functional for the aldehyde group of HOC-R molecules should be able to extrapolate to $\mathrm{R}$ groups outside of its training set. We tested this assumption by parametrizing aldehyde ${ }^{2} \Delta\left[{ }^{1} D\right]$ functionals using minimalbasis (STO-3G) HOC-R molecules with six different $\mathrm{R}$ groups: $\mathrm{H}, \mathrm{F}, \mathrm{OH}, \mathrm{CH}_{3}, \mathrm{Cl}$, and $\mathrm{OCH}_{3}$. Six different ${ }^{2} \Delta\left[{ }^{1} D\right]$ functionals were generated from this data. Each was trained 
on a data set that excluded data from one of the six $\mathrm{R}$ groups, and included half of the data from the other five groups. The functionals were tested for their ability to accurately model the aldehyde for both the five kinds of HOC-R molecules in the training set and the $R$ group excluded from the training set.

Details of the calculation are as follows. The $a b$ initio data set contained 250 calculations for each of the six kinds of HOC-R molecules. Each calculation had random geometric [60] and electrostatic 61] perturbations similar to those in the variable-geometry $(\mathrm{H}-\mathrm{H})_{5}$ chains above. Ab initio calculations were performed using MP2, as the different-sized HOC-R groups required a size-consistent method and full-CI was prohibitively expensive. The aldehyde ${ }^{2} \Delta\left[{ }^{1} D\right]$ functionals were fitted to the MP2 ${ }^{2} \Delta$ and the relaxed ${ }^{1} D[62]$ of the aldehyde group. The aldehyde functionals' performance was characterized by their ability to reproduce the "aldehyde correlation energy" defined as $E_{\text {corr }}^{H O C}=\sum\langle a c \mid b d\rangle{ }^{2} \Delta(a c, b d) ;\{a b c d\} \in$ HOC. All functionals used $40^{1} D$ and $30^{2} \Delta$ principal components. This was a significant dimensional reduction, as the aldehyde ${ }^{1} D$ and ${ }^{2} \Delta$ contain 65 and $\sim 2200$ degrees of freedom. Results from the six functionals are presented in Table VI. A plot of the extrapolation results is in Fig. 5 ,

In general, the results are quite good. ${ }^{2} \Delta\left[{ }^{1} D_{\text {exact }}\right]$ errors for the $\mathrm{R}$ groups in the training sets(Table VI, upper panel, off-diagonals) are small compared to both the average $E_{\text {corr }}^{H O C}$ $(-139.60 \mathrm{mH})$ and the standard deviation in $E_{\text {corr }}^{H O C}(13.30 \mathrm{mH})$. Most of the extrapolations

are also good, with ${ }^{2} \Delta\left[{ }^{1} D_{\text {exact }}\right]$ errors that are uniformly smaller than the corresponding ${ }^{2} \Delta\left[{ }^{1} D_{\text {avg }}\right]$ errors (diagonals of Table VI] compare upper and lower panels). The ${ }^{2} \Delta\left[{ }^{1} D_{\text {avg }}\right]$ energy errors are fairly good, as in the variable-geometry $(\mathrm{H}-\mathrm{H})_{5}$ systems, providing further evidence that the primitive, constant- ${ }^{2} \Delta$ functional works rather well for multiple geometries (see Sec. IIIB).

\section{DISCUSSION}

Nearsightedness and molecular similarity suggest that a rich data set of ab initio calculations on a functional group in various small molecules contains sufficient information to describe the functional group's behavior in large molecules. Here we explore new methods for generating semiempirical electronic structure models that are parametrized to such data sets. In particular, we consider a semiempirical, subsystem-based model of electron correlation. 
This model predicts the connected pair density matrix ${ }^{2} \Delta$ of molecular subsystems as a functional of the subsystem electron density matrix ${ }^{1} D$. Subsystem ${ }^{2} \Delta$ predictions are combined using a previously-developed divide-and-conquer-style treatment of the atomic-orbital-basis ${ }^{2} \Delta$ (LRDM). The ${ }^{2} \Delta$ functionals are used to obtain correlation-energy functionals for density functional theory (Eq. 9). The method is tested on chains of minimal-basis $(\mathrm{H}-\mathrm{H})_{5}$, which was treated as a system of four identical and overlapping $(\mathrm{H}-\mathrm{H})_{2}$ subsystems. The extrapolation abilities of ${ }^{2} \Delta\left[{ }^{1} D\right]$ functionals are tested on HOC-R molecules.

The $(\mathrm{H}-\mathrm{H})_{5}$ chain results demonstrate that the model works well for these simple systems. The results in Fig. 2 and Table [II] show that ${ }^{2} \Delta\left[{ }^{1} D\right]$ functionals fitted to ab initio data on $(\mathrm{H}-\mathrm{H})_{2}$ can reproduce the $(\mathrm{H}-\mathrm{H})_{2}{ }^{2} \Delta$ given the correct electron density matrix ${ }^{1} D_{\text {exact }}$. The ${ }^{2} \Delta$ of $(\mathrm{H}-\mathrm{H})_{5}$ systems can be modeled quite well using four overlaid $(\mathrm{H}-\mathrm{H})_{2}{ }^{2} \Delta\left[{ }^{1} D\right]$ functionals, as shown by the data in Fig. 3 and Table IV] Fig. 4 and Table $\nabla$ show that the ${ }^{2} \Delta\left[{ }^{1} D\right]$ functionals work reasonably well as DFT correlation functionals. These results are especially encouraging given the simple, linear form of the $v_{\text {corr }}\left[{ }^{1} D\right]$ functionals (Eq. 9). The subsystem ${ }^{2} \Delta\left[{ }^{1} D\right]$ functionals can extrapolate to molecules outside of the training set, as demonstrated by the HOC-R results in Sec. IIIE.

An interesting finding is that dimensional reduction of subsystem ${ }^{2} \Delta$ seems to be a reasonable approximation. All of the ${ }^{2} \Delta\left[{ }^{1} D\right]$ functionals had significant reductions in the dimensionality of ${ }^{2} \Delta$. This suggests that real molecular environments only explore a fraction of the total degrees of freedom in a functional group's ${ }^{2} \Delta$. This dimensional reduction may be useful for other models of electron correlation. Our results also suggest that, for these systems, simple quadratic functions are a fairly good model for the input:output relation of the dimensionally reduced data.

It is also interesting that subsystem ${ }^{2} \Delta\left[{ }^{1} D\right]$ functionals that are defined in a basis set can be used for multiple subsystem geometries. For both hydrogen chains and HOC-R molecules, a single ${ }^{2} \Delta\left[{ }^{1} D\right]$ functional provided good ${ }^{2} \Delta$ predictions for a fairly wide range of different geometries. Real-space ${ }^{2} \Delta\left[{ }^{1} D\right]$ functionals may be more general than those presented here. However, the success of the basis-set functionals is encouraging.

The utility of our semiempirical, subsystem-based treatment of electron correlation relies upon its ability to provide high-accuracy treatments of systems that are too large for current $a b$ initio methods. In particular, we would like to perform high-accuracy DFT calculations on polypeptides, using ${ }^{2} \Delta\left[{ }^{1} D\right]$ or ${ }^{2} D_{X C}\left[{ }^{1} D\right]$ functionals for the twenty most common amino 
acids. In order to provide an accurate treatment of electron correlation, each of the twenty functionals would be parametrized to high-level ab initio calculations in a large basis set (e.g. $\operatorname{CCSD}(\mathrm{T}) / \mathrm{cc}-\mathrm{pVTZ}$ ) 63]. These calculations would place each amino acid in a large number of small-molecule environments. The environments could include the amino acid in various 2- or 3-residue polypeptides, or with capping groups as in Ref. 9].

As stated above, the principle challenge to reaching this goal is the computational expense of parametrizing subsystem ${ }^{2} \Delta\left[{ }^{1} D\right]$ or ${ }^{2} D_{X C}\left[{ }^{1} D\right]$ functionals for moderately-sized subsystems in a large basis set. Parametrizing a ${ }^{2} \Delta\left[{ }^{1} D\right]$ functional for a group with $M$ basis functions requires $\mathcal{O}\left(\exp \left(M^{2}\right)\right)$ data points. In the current proof-of-concept work, we studied small subsystems in a minimal basis set, so that new ${ }^{2} \Delta\left[{ }^{1} D\right]$ functionals could be parametrized and tested in a relatively short time. Data sets for the systems studied here, minimal-basis $(\mathrm{H}-\mathrm{H})_{5}$ and HOC-R, could typically be generated in a couple of days on a single $2.8 \mathrm{GHz}$ Xeon processor.

Another challenge to using our approach for high-accuracy DFT on large systems is the computational expense of using the high-accuracy subsystem functionals, as this would require performing DFT calculations in a large basis set. One interesting possibility for mitigating this expense is to parametrize subsystem functionals that predict both ${ }^{1} D$ and ${ }^{2} \Delta$ in a large basis set as a function of ${ }^{1} D$ in a smaller basis. This would enable us to use a database of high-accuracy, large-basis-set subsystem calculations to correct small-basis-set DFT on a large system.

We believe that the general approach presented here (see flowchart, Sec. IIB) may be useful for modeling several aspects of electronic structure in addition to intra-subsystem electron correlation effects. We are currently developing a semiempirical, subsystem-based treatment of dispersion interactions in DFT that uses semiempirical functionals to predict a subsystem's polarizability as a function of its ${ }^{1} D$. Also, as mentioned above, we are developing a treatment of core polarization in effective core potentials. This treatment uses a semiempirical functional to predict the change in core electron density matrix as a function of the valence density matrix and the core-electron Hamiltonian.

This work explores a new approach for taking advantage of molecular similarity in electronic structure theory. The results suggest that it may be possible to construct accurate semiempirical models by extracting transferable information from ab initio data on small molecules. However, the applicability of the method to larger systems remains to be ex- 
plored.

The authors thank Craig J. Gallek for contributions to extensions to GAMESS for density matrix manipulation. This work was supported by the National Science Foundation. BGJ thanks the NSF for additional support. 


\section{SUPPORTING INFORMATION}

\section{A. Sources of error in fixed-geometry $(\mathbf{H}-\mathrm{H})_{5}$}

The conclusion that the error in fixed-geometry $(\mathrm{H}-\mathrm{H})_{5}$ is due to long-range order is supported by results from the $\left(C_{1}=7, C_{2}=8\right)^{2} \Delta\left[{ }^{1} D\right]$ functional discussed in Sec 【IB. This functional gave a better description of the fixed-geometry $(\mathrm{H}-\mathrm{H})_{2}$ systems than the functional in Table II. However, this functional does not give a better description of the $(\mathrm{H}-\mathrm{H})_{5}$ systems: the ${ }^{2} \Delta\left[{ }^{1} D_{\text {exact }}\right]\left|\delta E_{\text {corr }}\right|$ is 5.01 (11.30) $\mathrm{mH}$, much larger than the 1.81 (1.99) value in Table IV.

To further confirm that the fixed-geometry error is due to the effects of long-range order, we parametrized ${ }^{2} \Delta\left[{ }^{1} D\right]$ functionals for a new fixed-geometry $(\mathrm{H}-\mathrm{H})_{5}$ system with increased long-range order. This system was generated as in Sec. IIA but with an $(\mathrm{H}-\mathrm{H}) \leftrightarrow(\mathrm{H}-\mathrm{H})$ spacing of $1.0 \AA$ rather than $1.6 \AA$. Its $(\mathrm{H}-\mathrm{H})_{2}$ subsystems are expected to be even less similar to isolated $(\mathrm{H}-\mathrm{H})_{2}$ molecules. The increased long-range order is seen in an increased (though still quite small) subsystem decomposition error, with average (standard deviation) ${ }^{2} \Delta_{\text {xsub }}\left|\delta E_{\text {corr }}\right|$ of 1.01 (0.60) $\mathrm{mH}$ vs. the $0.02(0.04) \mathrm{mH}$ values in Table IV This system's ${ }^{2} \Delta\left[{ }^{1} \mathrm{D}\right](\mathrm{H}-\mathrm{H})_{2}$ functionals gave $\left|\delta E_{\text {corr }}\right|$ for $(\mathrm{H}-\mathrm{H})_{2}$ comparable to the values in Table III. average (standard deviation) values of the ${ }^{2} \Delta\left[{ }^{1} D_{\text {exact }}\right]\left|\delta E_{\text {corr }}\right|$ for the testing-set data are $0.29(0.43) \mathrm{mH}$. However, as expected, the increased long-range order meant that the ${ }^{2} \Delta\left[{ }^{1} D\right]$ functionals parametrized on isolated $(\mathrm{H}-\mathrm{H})_{2}$ molecules gave very poor results when applied to $(\mathrm{H}-\mathrm{H})_{5}$. The ${ }^{2} \Delta\left[{ }^{1} D_{\text {exact }}\right]\left|\delta E_{\text {corr }}\right|$ was 17.93 (4.89) $\mathrm{mH}$, much larger than the value in Table IV.

\section{B. Training set choice calculations for $(\mathbf{H}-\mathbf{H})_{2}{ }^{2} \Delta\left[{ }^{1} D\right]$ functionals}

The predictions of a semiempirical model should not depend on the choice of training set data. We tested this by parametrizing several different $(\mathrm{H}-\mathrm{H})_{2}{ }^{2} \Delta\left[{ }^{1} D\right]$ functionals (102 for variable-geometry systems, 74 for fixed-geometry systems), each with a different training set choice. Table VII presents the average and standard deviation, taken across the training set choices, of the average $\left|\delta E_{\text {corr }}\right|$ values of each data set.

To clarify how the results in Table VII were obtained, let $S_{\text {choice }}$ denote the set of $N_{\text {choice }}$ different choices of training set tested, where $N_{\text {choice }}$ equals 102 and 74 for variable- and 
fixed-geometry systems. The training set choices in $S_{\text {choice }}$ are indexed by $x$. Let $S_{\text {train }}^{x}$ denote the set of $(\mathrm{H}-\mathrm{H})_{2}$ molecules in training set $x$, where each molecule is indexed by $i_{x}$. Each $S_{\text {train }}^{x}$ contains 500 of the 1000 total $(\mathrm{H}-\mathrm{H})_{2}$ molecules (Table II), with the remainder in the test set. Let the absolute correlation energy error $\left|\delta E_{\text {corr }}\right|$ for ${ }^{2} \Delta\left[{ }^{1} D_{\text {exact }}\right]$ of each data point in training set $x$ be denoted $\left|\delta E_{\text {corr }}\right|\left(i_{x}\right)$, and let $A V E\{\}$ and $S T D E V\{\}$ denote the operations of calculating the average and standard deviation of a set of points. The average (standard deviation) values of the first entry in Table VII (row 1, column 2), denoted "A" and "B", are obtained as

$$
\begin{aligned}
& A=A V E\left\{A V E\left\{\left|\delta E_{\text {corr }}\right|\left(i_{x}\right), i_{x} \in S_{\text {train }}^{x}\right\}, x \in S_{\text {choice }}\right\} \\
& B=S T D E V\left\{A V E\left\{\left|\delta E_{\text {corr }}\right|\left(i_{x}\right), i_{x} \in S_{\text {train }}^{x}\right\}, x \in S_{\text {choice }}\right\}
\end{aligned}
$$

The results in Table VII verify that the ${ }^{2} \Delta\left[{ }^{1} D\right]$ functional predictions do not depend very much on the training set choice.

When parametrizing a model, it is useful to test models that were parametrized with an incorrect input:output relation in the training set. If the model is implemented correctly, and is modeling a real physical relationship, scrambling the data should degrade the results. Table VII includes results from a ${ }^{2} \Delta\left[{ }^{1} D\right]$ functional where the ${ }^{1} D$ from each molecule in the training set is paired randomly with the ${ }^{2} \Delta$ of a different molecule. This scrambles the input:output relation of the training data, and is denoted ${ }^{2} \Delta\left[{ }^{1} D_{\text {exact }}\right](\mathrm{scr})$. As expected, this functional is no better (and sometimes worse) than ${ }^{2} \Delta\left[{ }^{1} D_{\text {avg }}\right]$, which uses a single choice of ${ }^{2} \Delta$ for all data points. 
[1] Full-CI is exact within a given basis set.

[2] W. Kohn, Phys. Rev. Lett. 76, 3168 (1996).

[3] S. Goedecker, Rev. Mod. Phys. 71, 1085 (1999).

[4] L. Greengard and V.Rokhlin, J. Comp. Phys. 73, 325 (1987).

[5] C. A. White, B. G. Johnson, P. M. W. Gill, and M. Head-Gordon, Chem. Phys. Lett. 230, 8 (1994).

[6] C. A. White, B. G. Johnson, P. M. W. Gill, and M. Head-Gordon, Chem. Phys. Lett. 253, 268 (1996).

[7] W. Yang, Phys. Rev. Lett. 66, 1438 (1991).

[8] D. R. Bowler, T. Miyazaki, and M. J. Gillian, Journal of Physics: Condensed Matter 14 (2002).

[9] D. W. Zhang and J. Z. H. Zhang, J. Chem. Phys. 119, 3599 (2003).

[10] P. Pulay, Chem. Phys. Lett. 100, 151 (1983).

[11] S. Saebo and P. Pulay, Ann. Rev. Phys. Chem. 44, 213 (1993).

[12] C. Hampel and H.-J. Werner, J. Chem. Phys. 104, 6286 (1996).

[13] P.E.Maslen and M. Head-Gordon, Chem. Phys. Lett. 283, 102 (1998).

[14] G. E. Scuseria and P. Y. Ayala, J. Chem. Phys. 111, 8330 (1999).

[15] M. Schütz and H.-J. Werner, J. Chem. Phys. 114, 661 (2001).

[16] T. Van Voorhis and M. Head-Gordon, J. Chem. Phys. 115, 7814 (2001).

[17] S. Li, J. Ma, and Y. Jiang, J. Comp. Chem. 23, 237 (2002).

[18] N. Flocke and R. J. Bartlett, J. Chem. Phys. 118 (2003).

[19] K. Machida, Principles of molecular mechanics (Wiley, New York, 1999).

[20] J. Ridley and M. Zerner, Theoretica Chemica Acta 32, 111 (1973).

[21] E. J. Zoebisch, E. F. Healey, J. J. P. Stewart, and M. J. S. Dewar, JACS 107, 3902 (1985).

[22] A. Warshel and M. J. Karplus, JACS 94, 5612 (1972).

[23] F. Maseras and K. Morokuma, J. Comp. Chem. 16, 1170 (1995).

[24] F. Ercolessi and J. Adams, Europhysics Letters 26, 583 (1994).

[25] M. J. Mehl and D. A. Papaconstantopoulos, Phys. Rev. B 54, 4519 (1996).

[26] G. Tabacchi, C. J. Mundy, J. Hutter, and M. Parrinello, J. Chem. Phys. 117, 1416 (2002). 
[27] P. Tangney and S. Scandolo, J. Chem. Phys. 117, 8898 (2002).

[28] In a non-orthogonal basis like those used here, the real-space electron density ${ }^{1} D(\mathbf{r})$ and the real-space density matrix ${ }^{1} D\left(\mathbf{r}, \mathbf{r}^{\prime}\right)$ are obtained from ${ }^{1} D(a, b)$ as ${ }^{1} D(\mathbf{r})=$ $\sum_{a b} \phi_{a}^{*}(\mathbf{r}) \phi_{b}(\mathbf{r}){ }^{1} D(a, b)$ and ${ }^{1} D\left(\mathbf{r}, \mathbf{r}^{\prime}\right)=\sum_{a b} \phi_{a}^{*}(\mathbf{r}) \phi_{b}\left(\mathbf{r}^{\prime}\right){ }^{1} D(a, b)$.

[29] R. G. Parr and W. Yang, Density-Functional Theory of Atoms and Molecules (Oxford University Press, New York, 1989).

[30] D. A. Mazziotti, Phys. Rev. A 60, 4396 (1999).

[31] D. A. Mazziotti, Phys. Rev. A 60, 3618 (1999).

[32] P. Hohenburg and W. Kohn, Phys. Rev. 136, b864 (1964).

[33] W. Kohn and L. J. Sham, Phys. Rev. 140, A1133 (1965).

[34] K. Burke and J. P. Perdew, International Journal of Quantum Chemistry 56, 199 (1995).

[35] O. Gunnarson, M. Jonson, and B. I. Lundqvist, Phys. Rev. B 20, 3136 (1979).

[36] J. A. Alonso and L. A. Girfalco, Phys. Rev. B 17, 3735 (1978).

[37] S. L. Dixon and K. M. Merz, Jr., J. Chem. Phys. 104, 6643 (1996).

[38] T.-S. Lee, D. M. York, and W. Yang, J. Chem. Phys. 105, 2744 (1996).

[39] K. N. Kudin and G. E. Scuseria, Phys. Rev. B 61, 16440 (2000).

[40] N. N. amd K. Tada, S. Watanabe, H. Fujita, and K. Watanabe, Phys. Rev. Lett. 86, 540 (2001).

[41] B. G. Janesko and D. Yaron, J. Chem. Phys. 119, 1320 (2003).

[42] W. Kohn, Y. Meir, and D. E. Makarov, Phys. Rev. Lett. 80, 4153 (1998).

[43] Y. Andersson, D. C. Langreth, and B. I. Lundqvist, Phys. Rev. Lett. 76, 102 (1996).

[44] R. O. Jones and O. Gunnarson, Rev. Mod. Phys. 61, 689 (1989).

[45] D. C. Langreth and M. J. Mehl, Phys. Rev. B 28, 1809 (1983).

[46] A. D. Becke, J. Chem. Phys. 98, 5648 (1993).

[47] A. van de Wall and G. Ceder, Phys. Rev. B 59, 14992 (1999).

[48] W. Kohn and A. E. Mattsson, Phys. Rev. Lett. 81, 3487 (1998).

[49] R. Armiento and A. E. Mattsson, Phys. Rev. B 66, 165117 (2002).

[50] D. J. Tozer, V. E. Ingamells, and N. C. Handy, J. Chem. Phys. 105, 9200 (1996).

[51] Q. Zhao, R. C. Morrison, and R. G. Parr, Phys. Rev. A 50, 2138 (1994).

[52] O. V. Gritsenko, R. van Leeuwen, and E. J. Baerends, Phys. Rev. A 52, 1870 (1995).

[53] V. Cherkassky and F. Mulier, Learning from Data: Concepts, Theory, and Methods (Wiley- 
Interscience, 1998).

[54] G. Rauhut, J. W. Boughton, and P. Pulay, J. Chem. Phys. 103, 5662 (1995).

[55] M. Dolg, in Modern Methods and Algorithms of Quantum Chemistry, NIC Series 1, edited by J. Grotendorst (John Neumann Institute for Computing, 2000), pp. 479 - 508.

[56] The number of principle components required to describe an M-orbital ${ }^{1} D$ should scale asymptotically as $\mathcal{O}(M)$, but our subsystems are designed to be too small for this nearsighted assumption.

[57] The dimensionally-reduced subsystem blocks of ${ }^{2} \Delta_{P C A}$ are recombined using LRDM.

[58] M. W. Schmidt et al., J. Comput. Chem. 14, 1347 (1993).

[59] LRDM on minimal-basis $(\mathrm{H}-\mathrm{H})_{5}$ does not discard any ${ }^{2} \Delta$ information from the subsystem edges, as the subsystems are very small.

[60] Except for the central aldehyde carbon, the Cartesian coordinates of each atom in HOC-R were perturbed by a random variable $\delta$ where $|\delta| \leq 0.1 \AA$.

[61] 10 random fractional charges placed in a cube, $8.0 \AA$ to a side, centered on the aldehyde carbon atom with $\geq 1.2 \AA$ charge-atom separation.

[62] J. A. Pople, R. Krishnan, H. B. Schlegel, and J. S. Binkley, International Journal of Quantum Chemistry: Quantum Chemistry Symposium 13 (1979).

[63] T. H. Dunning, Jr., J. Chem. Phys. 90, 1007 (1989). 


\begin{tabular}{lccc} 
Approximation & ${ }^{2} \Delta_{\text {xsub }}{ }^{2} \Delta_{P C A}{ }^{2} \Delta\left[{ }^{1} D_{\text {exact }}\right]$ \\
\hline Subsystem decomposition & Yes & Yes & Yes \\
Dimensional reduction & No & Yes & Yes \\
Prediction from ${ }^{1} D$ & No & No & Yes
\end{tabular}

TABLE I: Types of connected pair density matrix ${ }^{2} \Delta$ obtained in the results, and the approximations associated with each. 


\begin{tabular}{lccccc} 
& \multicolumn{2}{c}{ Variable geometry } & \multicolumn{2}{c}{ Fixed geometry } \\
\cline { 2 - 3 } \cline { 5 - 6 } & $(\mathrm{H}-\mathrm{H})_{2}$ & $(\mathrm{H}-\mathrm{H})_{5}$ & & $(\mathrm{H}-\mathrm{H})_{2}$ & $(\mathrm{H}-\mathrm{H})_{5}$ \\
\hline$N_{d a t}$ & 1000 & 93 & 1000 & 99 \\
Point charges & 4 & 10 & 4 & 10 \\
\hline$(\mathrm{H}-\mathrm{H})$ bonds & $0.5 \leftrightarrow 1.0 \AA$ & & $0.7 \AA$ \\
$(\mathrm{H}-\mathrm{H}) \leftrightarrow(\mathrm{H}-\mathrm{H})$ & $0.9 \leftrightarrow 3.0 \AA$ & & $1.6 \AA$ \\
$C_{1}$ & 6 & & \multicolumn{2}{c}{4} \\
$C_{2}$ & \multicolumn{2}{c}{6} & & \multicolumn{2}{c}{5}
\end{tabular}

TABLE II: Details of the four data sets for linear dimerized hydrogen chains. $N_{\text {dat }}$ is the total number of molecules in the data set. $C_{1}$ and $C_{2}$ are the number of ${ }^{1} D$ and ${ }^{2} \Delta$ principal components used in the ${ }^{2} \Delta\left[{ }^{1} D\right]$ functionals (Eq. [7). 


\begin{tabular}{lcccc} 
Prediction & $\mathrm{V}$ train & $\mathrm{V}$ test & $\mathrm{F}$ train & $\mathrm{F}$ test \\
\hline${ }^{2} \Delta\left[{ }^{1} D_{\text {exact }}\right]$ & $1.95(1.61)$ & $1.91(1.85)$ & $0.37(0.51)$ & $0.46(0.96)$ \\
${ }^{2} \Delta_{P C A}$ & $1.29(1.29)$ & $1.27(1.22)$ & $0.11(0.16)$ & $0.13(0.27)$ \\
${ }^{2} \Delta\left[{ }^{1} D_{\text {ave }}\right]$ & $3.78(3.77)$ & $3.66(3.47)$ & $1.53(2.72)$ & $1.49(2.35)$ \\
$\mathrm{MP} 2$ & $85.83(18.93)$ & $68.52(2.04)$
\end{tabular}

TABLE III: Absolute $E_{\text {corr }}$ error $\left|\delta E_{\text {corr }}\right|(\mathrm{mH})$ for training and testing subsets of the variable- and fixed-geometry $(\mathrm{H}-\mathrm{H})_{2}$ subsystems ( $\mathrm{V}$ and F, respectively). Values are average (standard deviation) across the entire training or testing set, for a single choice of training set. MP2 $\left|\delta E_{\text {corr }}\right|$ values are included for comparison. The average and standard deviation of the correct $E_{\text {corr }}$ values are -114.39 (23.43) $\mathrm{mH}$ for the variable-geometry $(\mathrm{H}-\mathrm{H})_{2}$ and $-93.10(2.56) \mathrm{mH}$ for the fixed-geometry $(\mathrm{H}-\mathrm{H})_{2}$. 


\begin{tabular}{lcc} 
Prediction & $\mathrm{V}$ & $\mathrm{F}$ \\
\hline${ }^{2} \Delta\left[{ }^{1} D_{\text {exact }}\right]$ & $3.43(2.96)$ & $1.81(1.99)$ \\
${ }^{2} \Delta_{\text {xsub }}$ & $0.02(0.04)$ & $0.02(0.04)$ \\
${ }^{2} \Delta_{P C A}$ & $3.33(3.16)$ & $1.40(1.21)$ \\
${ }^{2} \Delta\left[{ }^{1} D_{\text {avg }}\right]$ & $9.21(6.53)$ & $2.55(5.06)$ \\
$\mathrm{MP} 2$ & $218.14(30.06)$ & $169.61(4.33)$
\end{tabular}

TABLE IV: Absolute $E_{\text {corr }}$ error $\left|\delta E_{c o r r}\right|(\mathrm{mH})$ for variable- and fixed-geometry $(\mathrm{H}-\mathrm{H})_{5}(\mathrm{~V}$ and F, respectively). Values are average (standard deviation) across the entire data set for the choice of training set used in Table III MP2 $\left|\delta E_{\text {corr }}\right|$ values are included for comparison. 


\begin{tabular}{ccc} 
System Prediction & $\mathrm{V}$ & $\mathrm{F}$ \\
\hline$(\mathrm{H}-\mathrm{H})_{2}{ }^{2} \Delta\left[{ }^{1} D_{H F}\right]$ & $5.72(3.91)$ & $3.21(1.02)$ \\
${ }^{2} \Delta\left[{ }^{1} D_{D F T}\right]$ & $4.10(3.34)$ & $1.24(0.66)$ \\
\hline$(\mathrm{H}-\mathrm{H})_{5}{ }^{2} \Delta\left[{ }^{1} D_{H F}\right]$ & $15.73(5.46)$ & $9.24(1.48)$ \\
${ }^{2} \Delta\left[{ }^{1} D_{D F T}\right]$ & $12.02(4.73)$ & $4.36(1.90)$
\end{tabular}

TABLE V: Absolute $E_{\text {corr }}$ error $\left|\delta E_{\text {corr }}\right|(\mathrm{mH})$ for DFT and corrected Hartree-Fock calculations using ${ }^{2} \Delta\left[{ }^{1} D\right]$ correlation energy functionals $\left({ }^{2} \Delta\left[{ }^{1} D_{H F}\right]\right.$ and ${ }^{2} \Delta\left[{ }^{1} D_{D F T}\right]$, respectively). Results are presented for $(\mathrm{H}-\mathrm{H})_{2}$ and $(\mathrm{H}-\mathrm{H})_{5}$, variable $(\mathrm{V})$ and fixed $(\mathrm{F})$ geometry hydrogen chains, average (standard deviation) over the entire data set for a single training set choice. 


\begin{tabular}{lcccccc} 
Excluded & $\mathrm{H}$ & $\mathrm{F}$ & $\mathrm{OH}$ & $\mathrm{CH}_{3}$ & $\mathrm{Cl}$ & $\mathrm{OCH}_{3}$ \\
\hline $\mathrm{H}$ & $\mathbf{2 . 5 6}$ & 1.05 & 1.11 & 1.17 & 1.09 & 1.10 \\
$\mathrm{~F}$ & 1.44 & $\mathbf{3 . 2 4}$ & 1.19 & 1.42 & 1.31 & 1.23 \\
$\mathrm{OH}$ & 1.33 & 1.42 & $\mathbf{1 . 4 2}$ & 1.50 & 1.21 & 1.47 \\
$\mathrm{CH}_{3}$ & 1.43 & 1.27 & 1.23 & $\mathbf{2 . 3 5}$ & 1.32 & 1.30 \\
$\mathrm{Cl}$ & 1.55 & 1.16 & 1.18 & 1.57 & $\mathbf{8 . 1 0}$ & 1.34 \\
$\mathrm{OCH}_{3}$ & 1.37 & 1.26 & 1.23 & 1.47 & 1.19 & $\mathbf{1 . 7 6}$ \\
Excluded & $\mathrm{H}$ & $\mathrm{F}$ & $\mathrm{OH}$ & $\mathrm{CH}$ & $\mathrm{Cl}$ & $\mathrm{OCH}$ \\
\hline $\mathrm{H}$ & $\mathbf{6 . 0 0}$ & 5.50 & 4.48 & 4.48 & 6.62 & 5.37 \\
$\mathrm{~F}$ & 6.07 & $\mathbf{5 . 4 4}$ & 5.09 & 4.80 & 6.47 & 4.88 \\
$\mathrm{OH}$ & 5.50 & 4.36 & $\mathbf{4 . 7 0}$ & 4.11 & 8.13 & 5.05 \\
$\mathrm{CH}_{3}$ & 6.37 & 5.83 & 4.76 & $\mathbf{4 . 6 9}$ & 6.88 & 5.52 \\
$\mathrm{Cl}$ & 5.01 & 4.82 & 4.85 & 4.28 & $\mathbf{9 . 0 9}$ & 5.09 \\
$\mathrm{OCH}_{3}$ & 5.67 & 5.17 & 4.74 & 4.35 & 7.70 & $\mathbf{5 . 1 2}$
\end{tabular}

TABLE VI: Absolute $E_{\text {corr }}^{H O C}$ errors $(\mathrm{mH})$ for the six different HOC-R ${ }^{2} \Delta\left[{ }^{1} D\right]$ functionals. The rows are the results for each of the six functionals, where the $\mathrm{R}$ group that was excluded from each functional's training data is listed in the first column. The columns show the mean absolute $E_{c o r r}^{H O C}$ error for each of the six kinds of HOC-R molecules in the testing set. Extrapolations to the $\mathrm{R}$ group excluded from each functional are shown in boldface. Upper and lower panels are data for ${ }^{2} \Delta\left[{ }^{1} D_{\text {exact }}\right]$ and ${ }^{2} \Delta\left[{ }^{1} D_{\text {avg }}\right]$. The extrapolation results for ${ }^{2} \Delta\left[{ }^{1} D_{\text {exact }}\right]$ are plotted in Fig. 5 , 


\begin{tabular}{lccc} 
System & ${ }^{2} \Delta\left[{ }^{1} D_{\text {exact }}\right]$ & ${ }^{2} \Delta\left[{ }^{1} D_{\text {avg }}\right]$ & ${ }^{2} \Delta\left[{ }^{1} D_{\text {exact }}\right]($ scr $)$ \\
\hline V train & $1.75(0.15)$ & $3.70(0.12)$ & $6.93(0.33)$ \\
V test & $1.83(0.15)$ & $3.75(0.13)$ & $7.00(0.39)$ \\
V (H-H $)_{5}$ & $3.12(0.23)$ & $10.18(0.69)$ & $12.91(0.74)$ \\
F train & $0.45(0.04)$ & $1.38(0.11)$ & $1.43(0.12)$ \\
F test & $0.48(0.03)$ & $1.36(0.15)$ & $1.41(0.08)$ \\
F $(\mathrm{H}-\mathrm{H})_{5}$ & $1.98(0.17)$ & $2.53(0.04)$ & $3.95(0.30)$
\end{tabular}

TABLE VII: Absolute $E_{\text {corr }}$ errors $\left|\delta E_{\text {corr }}\right|(\mathrm{mH})$ for $(\mathrm{H}-\mathrm{H})_{2}{ }^{2} \Delta\left[{ }^{1} D\right]$ functionals, for multiple choices of training set. Each entry is the average value of all molecules in the training or testing data set, average (standard deviation) over the training set choices (see text for details). Results are reported for variable- and fixed-geometry systems (respectively $\mathrm{V}$ and $\mathrm{F}$ ), for $(\mathrm{H}-\mathrm{H})_{2}$ training and testing sets and extrapolation to $(\mathrm{H}-\mathrm{H})_{5}$ (respectively train, test, and $\left.(\mathrm{H}-\mathrm{H})_{5}\right)$. 


\section{Figure captions}

FIG. 1: Schematic of a nearsightedness-based divide-and-conquer treatment of electronic structure for a generic four-element chain. The electronic structure of the three subsystems (boxed regions) are obtained separately (calculations I-III) and combined into an approximate electronic structure for the entire system ("Total"). The calculated electronic structure near the edges of each subsystem (dotted lines) is incorrect due to short-range edge effects, and is not used in the final approximate structure.

FIG. 2: Predicted vs. real $E_{\text {corr }}(\mathrm{mH})$ for variable- and fixed-geometry $(\mathrm{H}-\mathrm{H})_{2}(\mathrm{~A}$ and B). The correlation coefficient $\mathrm{R}^{2}$ between real and predicted $E_{\text {corr }}$ are in parentheses. To reduce congestion, the variable-geometry ${ }^{2} \Delta_{P C A}$ and ${ }^{2} \Delta\left[{ }^{1} D_{\text {ave }}\right] E_{\text {corr }}$ are shifted down by 30 and $60 \mathrm{mH}$. MP2 $E_{\text {corr }}$ are shifted down by 86 and $67 \mathrm{mH}$ for the variable- and fixed-geometry results, respectively.

FIG. 3: Predicted vs. real correlation energies $(\mathrm{mH})$ for variable- and fixed-geometry $(\mathrm{H}-\mathrm{H})_{5}(\mathrm{~A}$ and B), predicted using $(\mathrm{H}-\mathrm{H})_{2}$ functionals. $\mathrm{R}^{2}$ between real and predicted $E_{\text {corr }}$ are in parentheses. To reduce congestion, the variable-geometry ${ }^{2} \Delta_{x s u b},{ }^{2} \Delta_{P C A}$ and ${ }^{2} \Delta\left[{ }^{1} D_{a v e}\right] E_{\text {corr }}$ are shifted down by 60,60 , and $120 \mathrm{mH}$, respectively. MP2 $E_{\text {corr }}$ are shifted down by 216 and $163 \mathrm{mH}$ for the variable- and fixed-geometry results, respectively.

FIG. 4: Predicted vs. real correlation energies $(\mathrm{mH})$ for DFT and corrected Hartree-Fock calculations using $(\mathrm{H}-\mathrm{H})_{2}{ }^{2} \Delta\left[{ }^{1} \mathrm{D}\right]$ functionals. Results are presented for variable- and fixed-geometry $(\mathrm{H}-\mathrm{H})_{5}(\mathrm{~A}$ and $\mathrm{B}) . \mathrm{R}^{2}$ between real and predicted $E_{\text {corr }}$ are in parentheses. 
FIG. 5: Extrapolation results. Predicted vs. real ${ }^{2} \Delta\left[{ }^{1} D_{\text {exact }}\right] E_{\text {corr }}^{H O C}$ for the six kinds of HOC-R molecules. Each of the HOC-R data sets is modeled using the ${ }^{2} \Delta\left[{ }^{1} D\right]$ functional that was not trained on data from that $\mathrm{R}$ group. The correlation coefficients $\mathrm{R}^{2}$ between real and predicted $E_{\text {corr }}^{H O C}$ are in parentheses. Absolute $E_{\text {corr }}^{H O C}$ errors for the plotted data are the diagonal (boldface) entries in the upper panel of Table VI] 


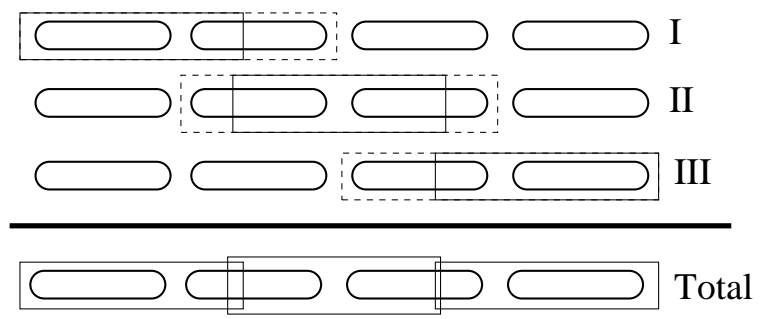

Figure 1

Janesko et. al.

Journal of Chemical Physics 

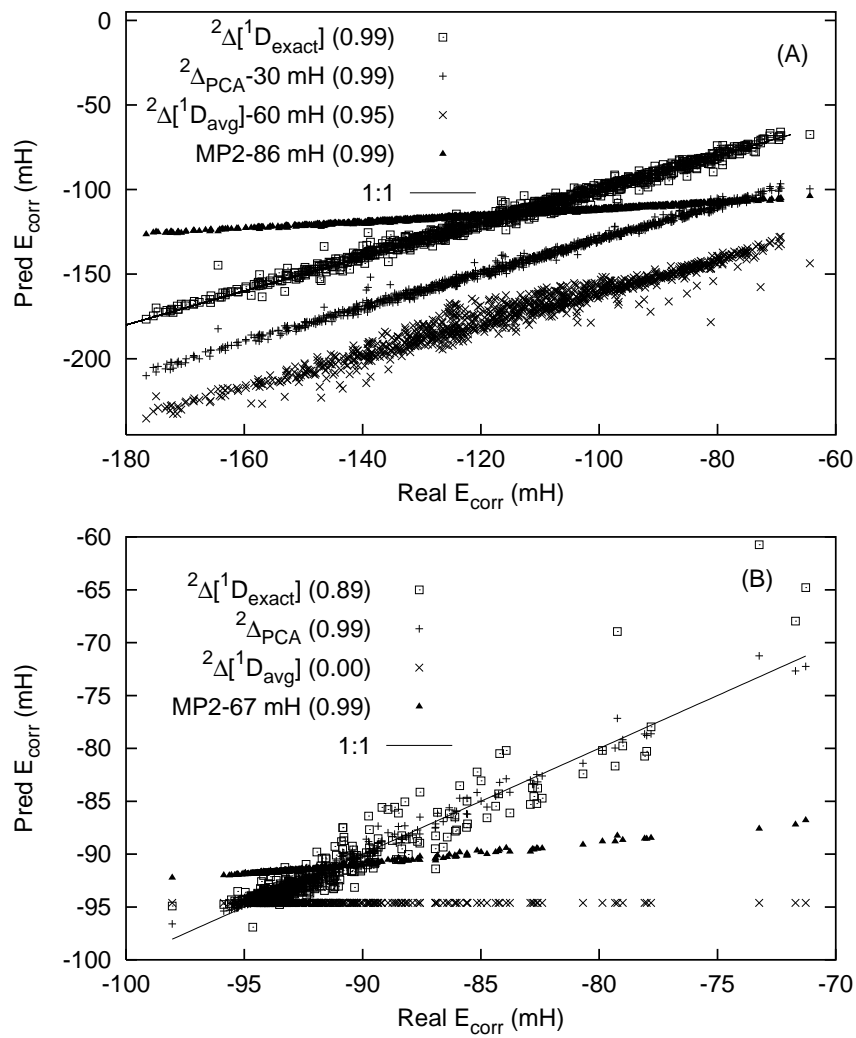

Figure 2

Janesko et. al.

Journal of Chemical Physics 

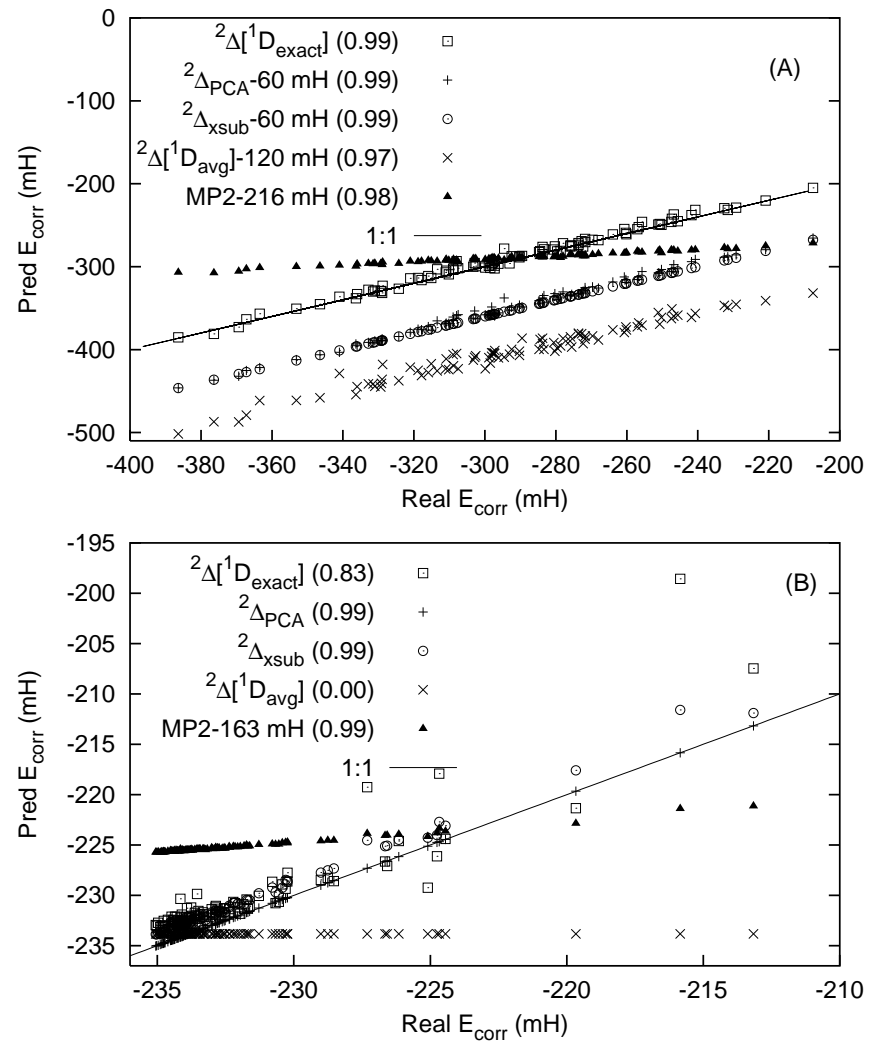

Figure 3

Janesko et. al.

Journal of Chemical Physics 

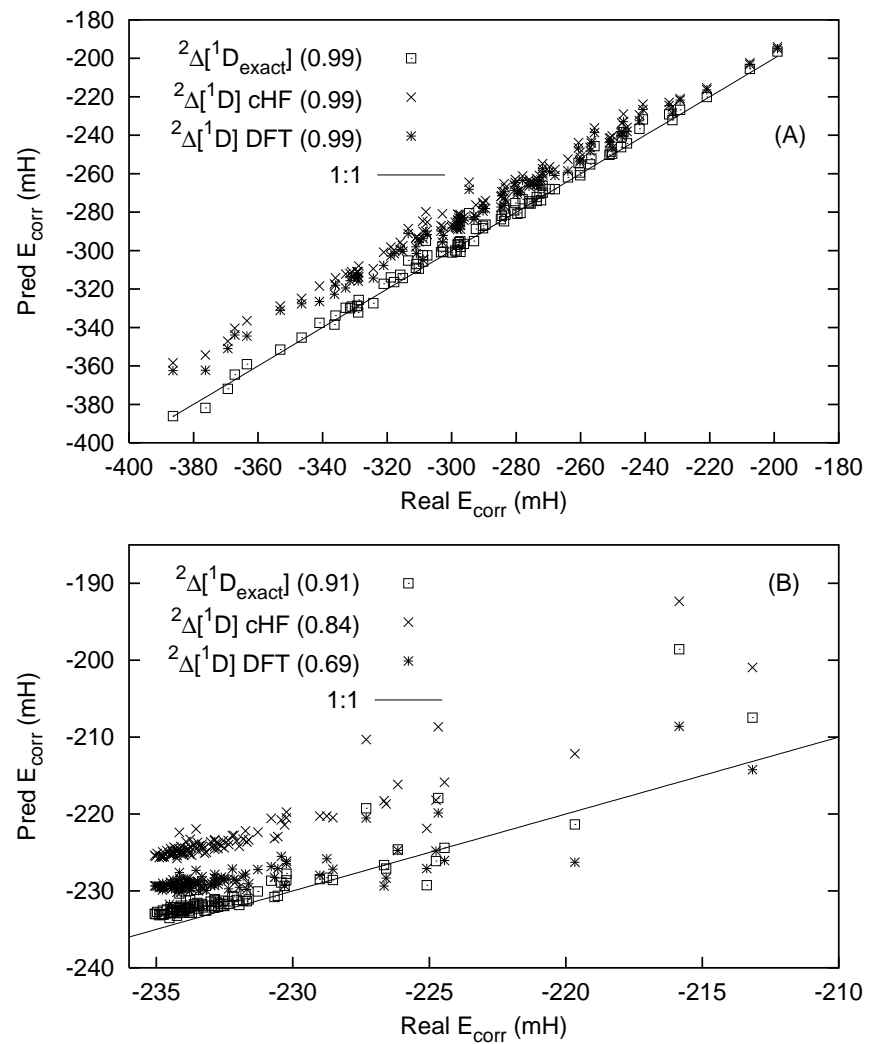

Figure 4

Janesko et. al.

Journal of Chemical Physics 


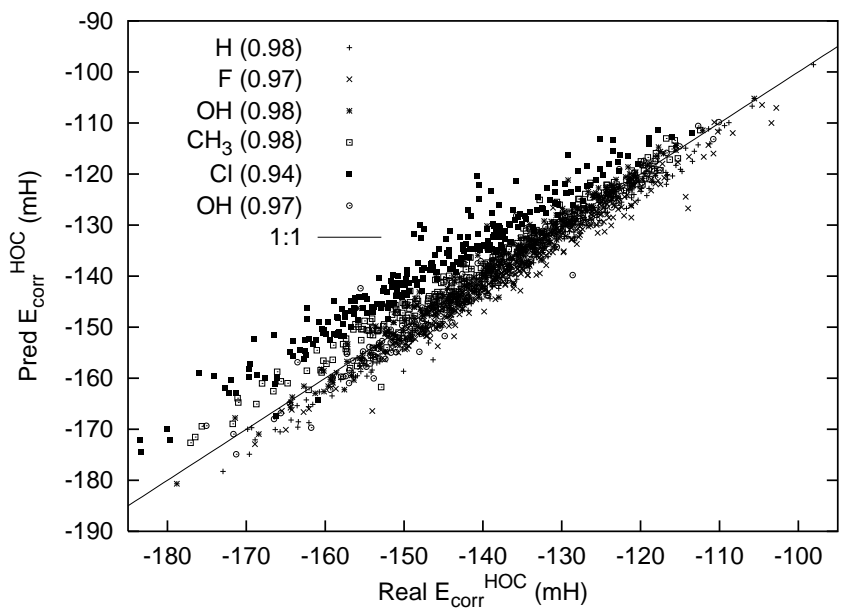

Figure 5

Janesko et. al.

Journal of Chemical Physics 